P133 (continued)

Results: Over 60\% of EFNEP staff perceived overall delivery is easier in urban environments. Over 50\% assumed urban areas would involve less travel and have an outpour of participants, however, traveling (20+ miles) was not uncommon to meet with groups with less than 10 in both rural and urban classes. Additionally, it was found that classes with $<5$ participants were meaningful and acceptable in rural environments, yet in urban environments, classes were oftentimes rescheduled due to low-participation rates ( $<5 /$ group). More than $70 \%$ stated difficulty in retaining $100 \%$ of class participants for the entire EFNEP lesson-series.

Conclusions and Implications: Extension agents have the responsibility to train EFNEP staff to meet the unique needs of urban and rural clients. Results from this research provides information for agents to adjust program planning, delivery, and staff training to aid educators to become more united as they understand the diversity between urban and rural counties and share how successes in these counties can bolster the Arizona's overall success in EFNEP.

Funding: Expanded Food and Nutrition Education Program.

\section{P134 Mutually Benefitting: Annual EFNEP Peer Lessons Increase EFNEP Staff Members' Knowledge and Teaching Skills}

Traci Armstrong Florian, MS, RD, tarmstro@cals.arizona. edu, The University of Arizona Cooperative Extension, 4341 East Broadway Road, Phoenix, AZ 85040

Objective: To demonstrate how EFNEP peer lessons strengthen group presentation skills and increase health-related knowledge among EFNEP paraprofessionals.

Design, Setting and Participants: At the beginning of the calendar year, peer lesson topics are chosen by staff and approved by the agent. The agent assigns the date of each presentation. The presentation must be a PowerPoint, 45-60 minutes in length, and information must come from reputable, research sources. The week before a presentation, the supervisor reviews it for content validity. At each peer lesson, the presenter is evaluated by each (peer) attendee. Evaluation comes in written and verbal form following a rubric. Once the written evaluations are viewed by the presenter and discussed with the supervisor, they are placed in the presenter's personnel file.

Outcome Measures and Analysis: A survey was designed and distributed to EFNEP staff. Data was entered in Microsoft Excel and analyzed.

Results: Nearly $82 \%$ of EFNEP staff reported an increase in nutritional knowledge with only $17 \%$ stating they already had the knowledge presented, and 76\% reported that the peer lesson was beneficial to their position and gave them knowledge to answer EFNEP clients' questions. The peer lesson concept is seen among staff as a positive way to learn and increase skills.
Conclusions and Implications: Research shows that professional development trainings are important to increase in knowledge and professionalism among staff and faculty (http://epa.sagepub.com/content/24/2/81. full.pdf + html). Results from this research shows that peer lessons are valued among EFNEP staff, increase knowledge, hone presentation skills, and are an effective manner to train staff.

Funding: Expanded Food and Nutrition Education Program.

\section{P135 Development and Validation of a General Nutrition Knowledge Questionnaire for Adults in Uganda}

RichardBukenya, MS, bukenya2@illinois.edu, University of Illinois at Urbana-Champaign, 1733 Melrose Village Circle, Apartment 1124, Urbana, IL 61801-0813; J. Muyonga, PhD, Makerere University Kampala, Uganda; J. Andrade, PhD, University of Illinois Urbana Champaign

Objective: At a basic level, nutrition knowledge is a determinant of nutrition behavior. The relevance of the content in a general nutrition knowledge questionnaire (GNKQ) targeting adult head teachers and health workers in Uganda as the first validation step was examined.

Design, Setting and Participants: The GNKQ comprised of 59 questions and structured into demographic information and six constructs assessing knowledge on recommendations, food groups, food choices, nutrition and disease relationship, food fortification, and sources of nutrition information. Five experts in the fields of health education, nutrition, agriculture and education reviewed the survey online using Qualtrics.

Outcome Measures and Analysis: The relevance, simplicity, ambiguity and clarity of questions were evaluated using a Likert scale (1-4). Experts were asked to recommend deletion/addition of items, change of language, foods and content, and its alignment with current nutrition policies in Uganda.

Results: The raw agreement proportion (RAP) for the whole survey on relevance was 0.89 (Gwet's AC1 $=0.75$, $\mathrm{P}<0.05)$. The RAP on relevance of questions was 0.85 (Gwet's $\mathrm{AC} 1=0.6, \mathrm{P}<0.05$ ) on recommendations; 0.88 (Gwet's $\mathrm{AC} 1=0.81, \mathrm{P}<0.05$ ) on food groups; 0.84 (0.62, $\mathrm{P}<0.05$ ) on food choices; 0.96 (Gwet's $\mathrm{AC} 1=0.91$, $\mathrm{P}<0.05)$ on nutrition and disease relationship; 0.73 (Gwet's AC1=0.23, P>0.05) on food fortification; and 0.9 (Gwet's $A C 1=0.7, P>0.05$ ) on sources of nutrition information. Experts recommended addition of more questions to the fortification construct.

Conclusions and Implications: All questions had "good" to "excellent" reliability agreement and were relevant to evaluate nutritional knowledge. Reviews are necessary to improve its clarity. Once validated, this selfadministered instrument will be used to gather nutrition knowledge among adults in Uganda.

Funding: USAID/ Borlaug Higher Education for Agricultural Research and Development (BHEARD). 